\title{
Novel chemiluminescent immunoassay to measure plasma aldosterone and plasma active renin concentrations for the diagnosis of primary aldosteronism
}

\author{
Kyoko Teruyama ${ }^{1} \cdot$ Mitsuhide Naruse $\mathbb{C}^{2,3} \cdot$ Mika Tsuiki $^{3} \cdot$ Hiroki Kobayashi ${ }^{4,5}$
}

Received: 15 April 2020 / Revised: 16 October 2020 / Accepted: 7 December 2020 / Published online: 9 February 2021

(c) The Author(s) 2021. This article is published with open access

\begin{abstract}
Determination of plasma aldosterone concentrations (PAC) and plasma active renin concentrations (ARC) is essential for the diagnosis of primary aldosteronism (PA). In Japan, although PAC and ARC are measured by radioimmunoassay and immunoradiometric assay, respectively, non-radioisotopic methods with better detection sensitivity, measurement accuracy, and technical simplicity are needed. We developed two-site sandwich chemiluminescent enzyme immunoassays (CLEIAs) to measure both PAC and ARC using monoclonal antibodies immobilized onto ferrite particles. The results of both assays are obtained simultaneously from a single plasma sample within $30 \mathrm{~min}$ using a fully automated system. The novel CLEIAs were validated using plasma samples from patients with PA $(n=52)$ and essential hypertension $(n=23)$. The PAC determined by the CLEIA was significantly correlated with that measured by liquid chromatography/mass spectrometry or conventional radioimmunoassay. The ARC determined by the CLEIA was significantly correlated with that measured by immunoradiometric assay. The limits of detection of the CLEIAs for PAC and ARC were $0.1 \mathrm{ng} / \mathrm{dl}$ and $0.04 \mathrm{pg} / \mathrm{ml}$, respectively, which were better than those of conventional methods (PAC: $2.5 \mathrm{ng} / \mathrm{dl}$; ARC: $5 \mathrm{pg} / \mathrm{ml}$ ). The PAC and PAC/ ARC ratio (ARR) were significantly higher, and the ARC significantly lower, in patients with PA than in those with essential hypertension. An ARR cut-off of $1.31 \mathrm{ng} / \mathrm{dl}$ per $\mathrm{pg} / \mathrm{ml}$ showed a sensitivity of $96.2 \%$ and specificity of $78.3 \%$ for PA screening. The newly developed CLEIAs for measuring PAC and ARC could provide a clinically powerful alternative to conventional methods used for hypertension screening in clinical practice.
\end{abstract}

\section{Introduction}

Primary aldosteronism (PA) is a major cause of secondary hypertension [1-5]. Considering the high prevalences of

Supplementary information The online version of this article (https:// doi.org/10.1038/s41371-020-00465-5) contains supplementary material, which is available to authorized users.

Mitsuhide Naruse

mtsnaruse@nifty.com

1 Marketing Department, Fujirebio Inc, Tokyo, Japan

2 Endocrine Center, Ijinkai Takeda General Hospital, Kyoto, Japan

3 Division of Endocrinology and Metabolism, NHO Kyoto Medical Center, Kyoto, Japan

4 Division of Nephrology, Hypertension, and Endocrinology, Nihon University School of Medicine, Tokyo, Japan

5 Section on Genetics and Epidemiology, Research Division, Joslin Diabetes Center, Boston, MA, USA cerebrovascular and cardiovascular complications in patients with PA compared with patients with essential hypertension (EH) [5-7], early diagnosis of and specific treatments for hyperaldosteronism are essential. Determination of the plasma aldosterone concentration (PAC) and plasma renin activity (PRA) is indispensable for diagnosing PA, including screening, confirmatory testing, and subtype diagnosis by adrenal venous sampling according to clinical practice guidelines [8-10]. Since measurement of these markers by liquid chromatography/tandem mass spectrometry (LC-MS/MS) [11] is not indicated in Japan because of the national health insurance system [12] designating the cost of each laboratory investigation, radioimmunoassay (RIA) has been used to determine the PAC, ARC, and PRA in clinical practice. Various issues with RIAs, such as the usage and disposal of radioisotopic materials, complexity of the manual assay, poor traceability of certified reference materials, and low detection sensitivity at lower concentrations [13, 14], remain to be resolved.

We developed new CLEIAs for detecting PAC and plasma ARC characterized by high sensitivity, sufficient 
traceability back to the certified reference materials, and versatile and efficient implementation by an automated system.

\section{Subjects and methods}

\section{Patients}

Patients referred to Kyoto Medical Center for further investigation of hypertension were evaluated. Diagnostic criteria for diagnosing PA is shown in Supplementary. In short, PA was diagnosed if the screening test was positive and at least one confirmatory test showed positive results. $\mathrm{EH}$ was diagnosed if any secondary cause of hypertension was excluded. The numbers of patients with PA, EH, and other miscellaneous diseases were 52, 23, and 33, respectively. Other miscellaneous diseases include renovascular hypertension, non-functioning adenoma, pheochromocytoma, paraganglioma, ACTH-independent macronodular adrenal hyperplasia, subclinical Cushing syndrome, and adrenocortical carcinoma were used for method comparison using peripheral blood. Apart from peripheral blood, we also evaluated the level of PAC and ARC by RIA and CLEIA using 50 samples from 5 patients for adrenal vein blood, and 18 samples from 18 patients for urine, respectively.

As part of routine clinical investigations, blood samples were collected into EDTA-2Na tubes from the antecubital veins of patients in the sitting position for at least $30 \mathrm{~min}$ in the clinic. Plasma samples were stored at less than $-20^{\circ} \mathrm{C}$ and subjected to our CLEIA immediately after thawing to room temperature in a $20^{\circ} \mathrm{C}$ water bath. In addition, plasma samples collected from the adrenal veins of patients with PA $(n=50)$ and urine samples collected from patients with hypertension $(n=18)$ were analyzed.

The study was conducted according to the tenets of the Declaration of Helsinki. All patients provided written informed consent. The study was approved by the ethical committee of Kyoto Medical Center (\#17-105).

\section{Development of new CLEIAs}

Our CLEIAs for measuring PAC and ARC were developed using two monoclonal antibodies against different epitopes of aldosterone (Fujirebio, Inc., Tokyo, Japan) and renin (Fujirebio, Inc.), respectively. The limit of detection (LoD), accuracy, precision, linearity, and recovery were determined for each CLEIA according to the recommendations of the Clinical and Laboratory Standards Institute (EP17-A2, EP05-A3, EP06-A). In addition, using the dedicated fully automated systems (LUMIPULSE ${ }^{\circledast}$ Presto II and LUMIPULSE ${ }^{\circledast}$ L2400) with CLEIAs enables simultaneous determination of PAC and ARC, respectively, from a single plasma sample, requiring $\sim 20 \mathrm{~min}$.

\section{CLEIA for PAC}

To detect PAC, we developed a two-site sandwich immunoassay using two specific monoclonal antibodies (primary antibody: anti-aldosterone mouse monoclonal antibody; secondary antibody: anti-metatype chicken antibody recognizing the aldosterone-aldosterone monoclonal antibody complex) against different epitopes of aldosterone. The plasma sample $(30 \mu \mathrm{l})$ was incubated with $250 \mu \mathrm{l}$ reagent A containing the primary antibody immobilized onto ferrite particles and incubated for $10 \mathrm{~min}$ at $37^{\circ} \mathrm{C}$. After washing with magnetic beads, the sample was incubated with $250 \mu \mathrm{l}$ reagent B containing the ALPconjugated secondary antibody and incubated for $10 \mathrm{~min}$ at $37^{\circ} \mathrm{C}$. After washing the magnetic beads, the complex consisting of the primary antibody, ALP-conjugated secondary antibody, and ferrite particles was dispersed, and $200 \mu \mathrm{l}$ solution containing 3-(2'-spiroadamantane)4-methoxy-4-(3"-phosphoryloxy) phenyl-1, 2-dioxetane disodium salt (AMPPD) was added to measure chemiluminescence [15]. The urine aldosterone concentration was determined following the same methods after deconjugation of glucuronic acid by $\mathrm{HCl}$ and neutralization. The CLEIA for PAC was calibrated in-house using a certified reference material for aldosterone [16]. (Fig. S1). Interference with assays measuring ascorbic acid, hemoglobin, bilirubin, bilirubin-conjugate, chyle, and rheumatoid factor, as well as cross-reactivity with corticosterone, cortisol, dexamethasone, spironolactone, progesterone, and 18-hydroxycorticosterone were analyzed. The accuracy, precision, linearity, and recovery of the CLEIA were measured.

\section{Novel CLEIA for ARC}

For ARC detection, we developed two-site sandwich immunoassay using two specific mouse monoclonal antibodies against different epitopes of renin. An anti-active renin mouse monoclonal antibody that binds to the active site of active renin specifically was used as the detection antibody. An anti-renin monoclonal antibody that recognizes the non-active site of renin was used as the capture antibody. The plasma sample $(40 \mu \mathrm{l})$ was incubated with $250 \mu \mathrm{l}$ reagent A containing the detection antibody immobilized to ferrite particles and incubated for $10 \mathrm{~min}$ at $37^{\circ} \mathrm{C}$. After washing with magnetic beads, the sample was incubated with $250 \mu$ reagent B containing the ALP-conjugated capture antibody and incubated for $10 \mathrm{~min}$ at $37^{\circ} \mathrm{C}$. After washing the magnetic beads, the complex consisting of the detection antibody, ALP-conjugated capture antibody, and 
ferrite particles was dispersed, and $200 \mu$ l solution containing AMPPD was added to measure chemiluminescence. The CLEIA for ARC was calibrated using in-house human recombinant renin (human activated renin [GenBank accession number NM_000537], amino acids 67-406 with C-terminal HIS tag) as the reference material. The accuracy, precision, linearity, and recovery of the CLEIA were measured.

\section{Correlations between the novel CLEIA and conventional assays}

Correlation and linear regression analyses were conducted to compare PAC measurements by our novel CLEIA with those by LC-MS/MS and a conventional RIA (SPAC-S Aldosterone kits, Fujirebio Inc.), and to compare plasma ARC measurements by our novel CLEIA with those by a conventional immunoradiometric assay (IRMA) (Renin IRMA FR, Fujirebio Inc.) and PRA by a conventional RIA (Renin activity FR, Fujirebio Inc.), respectively.

\section{Clinical validation of the novel CLEIAs}

PAC, ARC, and the PAC/ARC ratio (ARR) determined by the CLEIAs were compared between patients with PA and patients with EH.

\section{Statistical analysis}

PAC, ARC, and ARR were compared between the patient groups using the Mann-Whitney $U$ test. In the method comparison study, regression analysis was performed using the Passing-Bablok method and Pearson's correlation coefficients. Bland-Altman analysis was used to evaluate mean differences. The statistical significance was set at $P \leq$ 0.05. Statistical analyses were performed using Analyse-it software (Analyse-it, Ltd.) and SAS for Windows (version 9.4; SAS institute, Cary, NC).

\section{Results}

\section{CLEIA for PAC}

Three different concentrations of certified reference materials for ensuring the traceability of aldosterone (NMIJ CRM 6402-a) were assayed in duplicate using our CLEIA for PAC. The certainty of the CLEIA for measuring the certified reference materials ranged from 99 to $104 \%$ (Table 1).

The LoD of the CLEIA for PAC was $0.1 \mathrm{ng} / \mathrm{dl}$, and the upper limit of the measurement range was $200 \mathrm{ng} / \mathrm{dl}$. There was no significant cross-reactivity of the CLEIA with
Table 1 Traceability of the CLEIA against the certified reference materials of aldosterone with three different concentrations.

\begin{tabular}{llll}
\hline & $\begin{array}{l}\text { Certified } \\
\text { value } \\
\text { (ng/dL) }\end{array}$ & $\begin{array}{l}\text { Aldosterone } \\
\text { concentration by the } \\
\text { CLEIA (ng/dL) }\end{array}$ & $\begin{array}{l}\text { Certainty of the } \\
\text { CLEIA assay }\end{array}$ \\
\hline CRM Level1 & 20.1 & 20.99 & $104 \%$ \\
CRM Level2 & 41.1 & 40.67 & $99 \%$ \\
CRM Level3 & 79.2 & 80.49 & $102 \%$ \\
\hline
\end{tabular}

The value of the certified reference material of aldosterone (NMIJ CRM 6402-a) was confirmed. The recovery ratio by CLEIA against its certified value were $99-104 \%$.

corticosterone, cortisol, dexamethasone, spironolactone, progesterone, or 18-hydroxycorticosterone. There was no significant interference with the assays measuring ascorbic acid, hemoglobin, bilirubin, bilirubin-conjugate, chyle, or rheumatoid factor. The accuracy was 94-98\% (Table S1a), and the coefficient of variation, as an indicator of the precision, was 1.7-3.0\% (Table S2a). The dilution linearity of the samples with concentrations differing from the expected value was $96-104 \%$ (Table S3a). The recovery rate was 97-101\% (Table S4a).

The PAC in the peripheral blood measured by the CLEIA was significantly correlated with the PAC measured by LC-MS/MS $(y=1.0 \mathrm{x}-0.73, r=0.996, p<0.01 ; n=41)$ (Fig. 1a). There was a significant correlation between the results of the two assay methods even for PACs $<10 \mathrm{ng} / \mathrm{dl}$. The mean difference was -0.573 (95\% Limits of agreement [LoA]: -6.53 to 5.38) (Fig. 1b). Although the PAC measured by the CLEIA showed a significant correlation with that measured by conventional RIA $(y=0.62 \mathrm{x}-1.98, r=$ $0.979, p<0.01 ; n=132$ ) (Fig. 1c), the correlation between the CLEIA and conventional RIA results was weaker than that between the CLEIA and LC-MS/MS results. The correlation was especially weaker when the PAC was $<10 \mathrm{ng} / \mathrm{dl}$ $(y=0.39 \mathrm{x}-0.19, r=0.634, p<0.01 ; n=86)$. To validate the CLEIA measurements, the PAC of two discrepant samples A and B in Fig. 1c, was determined by LC-MS/MS. The LC-MS/MS result was similar to the CLEIA result (Table 2), with a mean difference of -11.4 (95\% Limits of agreement [LoA]: -31.8 to 9.04) (Fig. 1d).

PAC in the adrenal vein blood measured by the CLEIA was significantly correlated with that measured by conventional RIA. Although PAC in the adrenal vein blood measured by the CLEIA was significantly correlated with the PAC measured by RIA, the obtained values were higher in the RIA than CLEIA $(y=0.84 \mathrm{x}-51.2, r=$ $0.869, p<0.01 ; n=50$ ) (Fig. 2a). The PAC of sample C in Fig. 2a, which showed a significant difference between the CLEIA and RIA (7453.3 vs. $2120 \mathrm{ng} / \mathrm{dl}$, respectively), measured $7462.9 \mathrm{ng} / \mathrm{dl}$ by LC-MS/MS. The urine aldosterone concentration measured by the CLEIA 

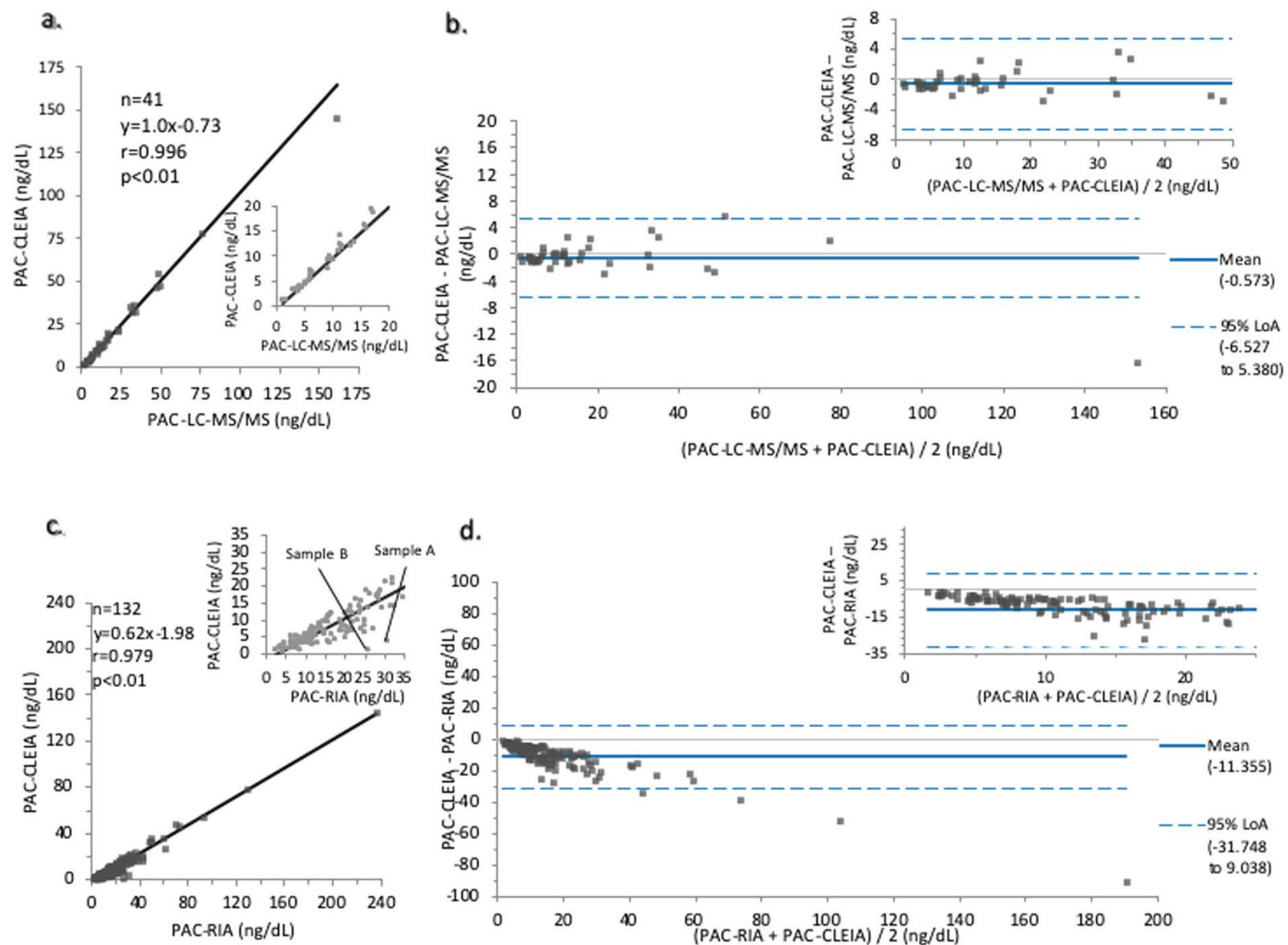

Fig. 1 Correlations of the plasma aldosterone concentration (PAC) in peripheral blood measured by chemiluminescent enzyme immunoassay (CLEIA). Correlations between CLEIA and liquid chromatography/ mass spectrometry (LC-MS/MS) (a). Bland-Altman analysis was performed to analyze the correlations of the CLEIA results with those of LC-MS/MS (b). Correlations of the plasma aldosterone concentration (PAC) in peripheral blood measured by

Table 2 Comparison of PAC between three assay methods in two samples with dissociated results between CLEIA and RIA.

\begin{tabular}{llll}
\hline Sample no. & CLEIA (ng/dL) & RIA (ng/dL) & LC-MS/MS (ng/dL) \\
\hline Sample A & 3.36 & 30.8 & 4.0 \\
Sample B & 0.76 & 26.0 & 1.17
\end{tabular}

Sample A and B showed discrepant results between CLEIA and RIA in Fig. 1c. These samples were measured with LC-MS/MS and compared to the value of RIA and CLEIA in Table 2. CLEIA and LCMS/MS showed similar values.

was correlated significantly with that measured by RIA (Fig. 2b).

\section{CLEIA for ARC}

The LoD of the CLEIA for ARC was $0.04 \mathrm{pg} / \mathrm{ml}$, and the upper limit of the measurement range was $1000.00 \mathrm{pg} / \mathrm{ml}$, chemiluminescent enzyme immunoassay (CLEIA) with that measured by radioimmunoassay (RIA) (c). The PAC in samples A and B showed significant discrepancy between the CLEIA and RIA measurements and was measured by LC-MS/MS as well. Bland-Altman analysis was performed to analyze the correlations of the CLEIA results with those of RIA (d). The correlation between the three methods was investigated by Passing-Bablok regression analysis.

respectively. The accuracy of the CLEIA was 100-106\% (Table S1b), and the coefficient of variation, as an indicator of the precision, was $3.0-4.1 \%$ (Table S2b). The dilution linearity of the samples with concentrations differing from the expected value was $97-104 \%$ (Table S3b). The recovery rate was $93-97 \%$ (Table S4b). The ARC measured by the CLEIA was significantly correlated with that measured by IRMA $(y=1.0 \mathrm{x}-0.31, \quad r=1.00, \quad p<0.01 ; \quad n=50)$ (Fig. 3a). The mean difference was 0.636 (95\% Limits of agreement [LoA]: -5.83 to 7.10 ) (Fig. $3 b$ ). The ARC measured by the CLEIA was significantly correlated with the PRA measured by RIA $(y=7.2 \mathrm{x}-1.55, r=0.920, p<$ $0.01 ; n=53$ ) (Fig. 3c).

\section{Clinical validation of the CLEIAs for PAC and ARC}

The CLEIA PAC and plasma ARC measurements were compared between the patients with PA and those with $\mathrm{EH}$. 
Fig. 2 Conrrelations of the plasma aldosterone concentration (PAC) in adrenal vein blood or urine. Correlations of the PAC in adrenal vein blood (a) or the urine aldosterone concentration (b) between CLEIA and RIA. The correlation between the two methods was investigated by Passing-Bablok regression analysis.

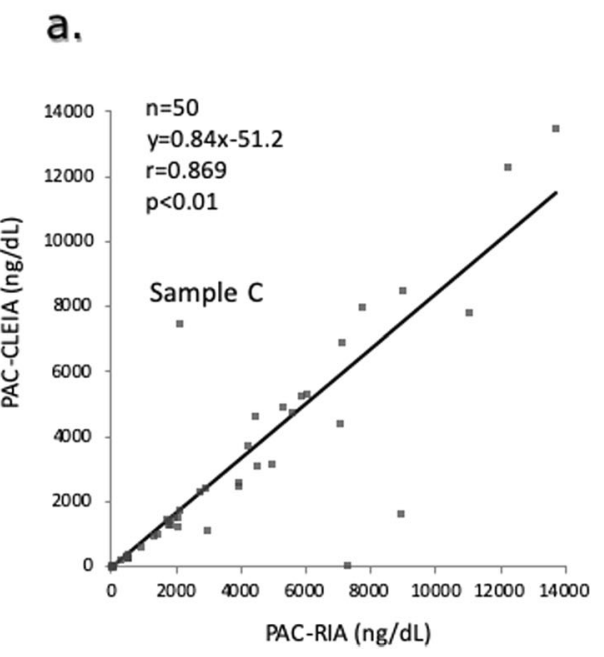

b,

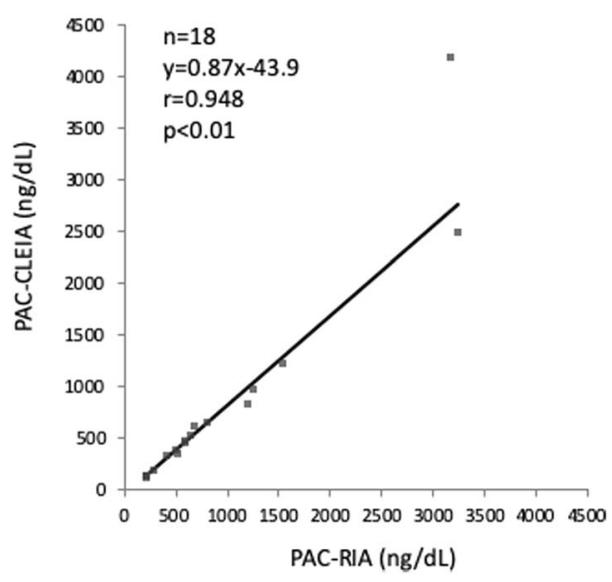

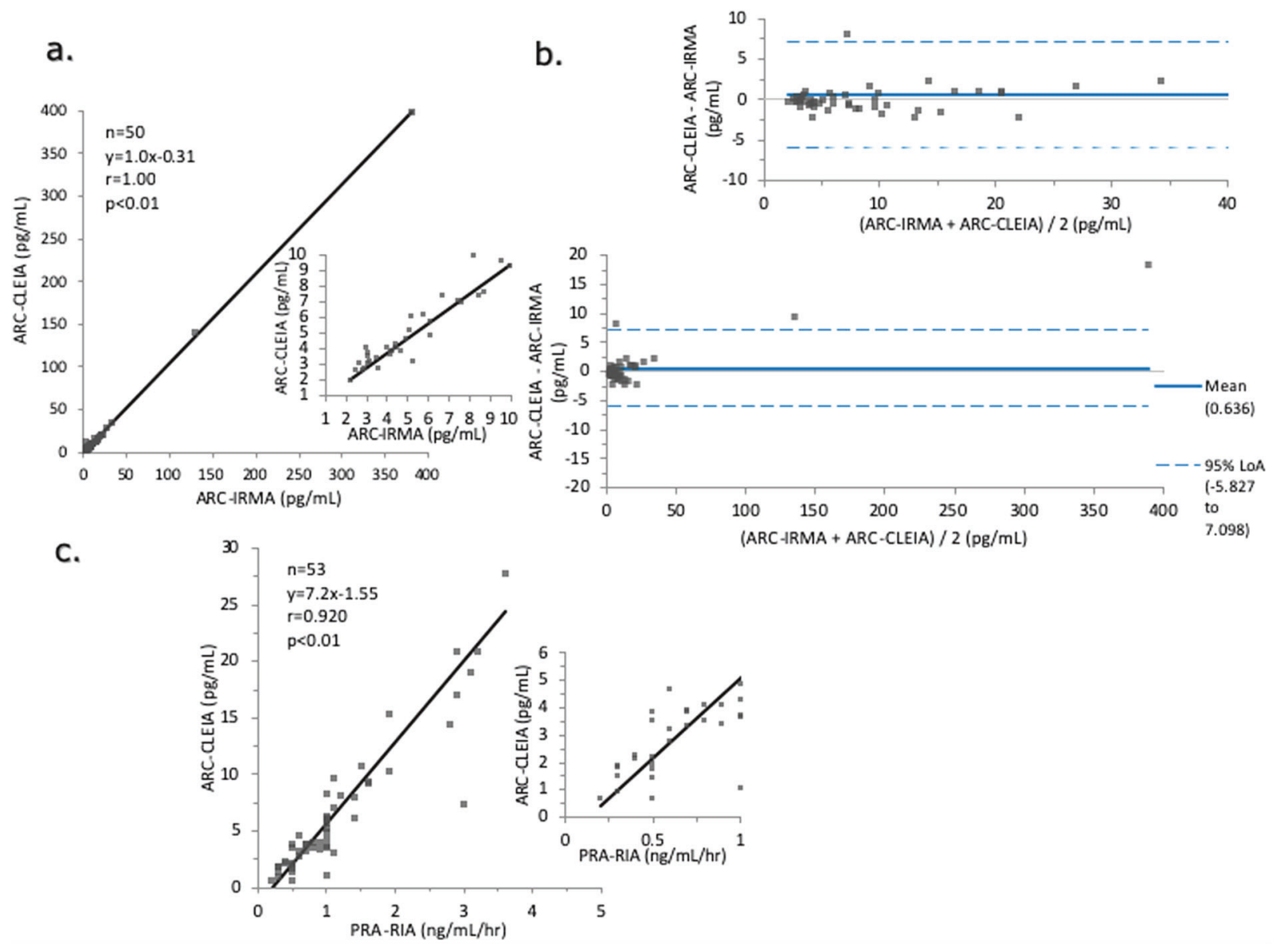

Fig. 3 Correlation of the plasma active renin concentration (ARC) and plasma renin activity (PRA). Correlation of the ARC in the peripheral blood between chemiluminescent enzyme immunoassay (CLEIA) and immunoradiometric assay (IRMA) (a). Bland-Altman analysis was performed to analyze the correlation between the ARC

The baseline clinical characteristics of the patients are shown in Table 3. The PAC was significantly higher, and the plasma ARC was significantly lower, in patients with measured by the CLEIA and that measured by IRMA (b). Correlations of the plasma ARC and PRA in the peripheral blood between the CLEIA and radioimmunoassay (RIA) (c). The correlation between the two methods was investigated by Passing-Bablok regression.

PA (PAC: $13.1 \mathrm{ng} / \mathrm{dl}$; ARC: $3.7 \mathrm{pg} / \mathrm{ml}$ ) than in patients with EH (PAC: $5.2 \mathrm{ng} / \mathrm{dl}$; ARC: $7.1 \mathrm{pg} / \mathrm{ml}$ ) (Fig. $4 \mathrm{a}, \mathrm{b}$ ). The plasma ARC measured by the CLEIA in all patients with 
PA above $0.2 \mathrm{pg} / \mathrm{ml}$, which was higher than the $0.2 \mathrm{pg} / \mathrm{ml}$ analytic sensitivity of the CLEIA.

The ARR measured by the CLEIA was significantly higher in patients with PA $(3.31 \mathrm{ng} / \mathrm{dl}$ per $\mathrm{pg} / \mathrm{ml})$ than in patients with EH $(0.52 \mathrm{ng} / \mathrm{dl}$ per pg/ml) (Fig. 4c). Receiver operating characteristic (ROC) analysis was performed to investigate the diagnostic value of the ARR for distinguishing PA from EH. The optimal ARR cutoff for discriminating PA from EH was $1.31 \mathrm{ng} / \mathrm{dl}$ per $\mathrm{pg} / \mathrm{ml}$, which yielded an area under the ROC curve of 0.967 (95\% CI: 0.932-1.000), sensitivity of $96.2 \%$, and specificity of $78.3 \%$ (Fig. 5 ).

In this study, the conversion formulas between conventional and the new assays were as follows: PAC (CLEIA) $=$ $0.62 \times$ PAC $($ RIA $)-1.98$, and PAC $($ CLEIA $) / P R A ~(R I A)=$

Table 3 Baseline characteristics of patients with PA and EH.

\begin{tabular}{lll}
\hline & PA $(n=52)$ & EH $(n=23)$ \\
\hline Age (years) & $61(52-68)$ & $59(49-73)$ \\
Sex, males/females & $24 / 51$ & $11 / 17$ \\
$\begin{array}{l}\text { Body mass index }\left(\mathrm{kg} / \mathrm{m}^{2}\right) \\
\text { Systolic blood }\end{array}$ & $129(21.2-26.3)$ & $25.3(23.6--28.7)$ \\
pressure (mmHg) & & $133(125-145)$ \\
$\begin{array}{l}\text { Diastolic blood } \\
\text { pressure }(\mathrm{mmHg})\end{array}$ & $79(71-90)$ & $76(71-90)$ \\
eGFR (mL/min/1.73 m²) & $62.8(54.0-73.9)$ & $69.4(62.4-84.5)$ \\
$\begin{array}{l}\text { Baseline PAC }(\mathrm{ng} / \mathrm{dL}) \\
\text { Baseline PRA }(\mathrm{ng} / \mathrm{mL} / \mathrm{h})\end{array}$ & $0.8(0.4-1.0)(n=24)$ & $1.1(1.0-1.9)(n=13)$ \\
Baseline ARC $(\mathrm{pg} / \mathrm{mL})$ & $3.70(2.10-5.92)$ & $7.05(3.61-9.69)$ \\
\hline
\end{tabular}

Continuous data are shown as the median (interquartile range).

$P A$ primary aldosteronism, $E H$ essential hypertension, $e G F R$ estimated glomerular filtration rate, $P A C$ plasma aldosterone concentration, $P R A$ plasma renin activity, $A R C$ active renin concentration.
$-1.05+0.49 \times$ PAC (RIA)/PRA (RIA). For example, the ARR cut-off value of 20 determined by RIA for PAC and PRA, which is used to screen for PA, can be converted to 8.75 by PAC (CLEIA)/PRA (RIA). The PAC cut-off value of 6 determined by RIA, which is used to diagnose PA by the saline infusion test, can be converted to 1.74 by PAC (CLEIA).

\section{Discussion}

The prevalence of PA has reached $5-10 \%$ in the hypertensive population $[9,17,18]$. Since patients with PA are more likely to experience cardiovascular and cerebrovascular diseases compared with patients with EH [19-22], an early and accurate diagnosis of PA is of great importance. However, there are methodological issues with RIAs for measuring PAC, ARC, and PRA, which are critical for the screening and diagnosis of PA. In this study, we developed novel CLEIAs for measuring PAC, urine aldosterone concentration, and plasma ARC. These CLEIAs demonstrated good traceability to the certified reference material of aldosterone, good linearity over a wide range of concentrations, and good correlation with the LC-MS/MS results both in plasma and urine samples.

These findings agree with previous reports of the advantages of CLEIAs [23-25]. Japan's health and medical services include a universal health insurance system that covers the medical costs of all citizens. However, the cost of LC-MS/MS is not covered by national insurance. Hence, RIA is the only assay method for PAC covered by Japanese health insurance; yet, this assay has demonstrated limitations in sensitivity and reproducibility, especially at lower
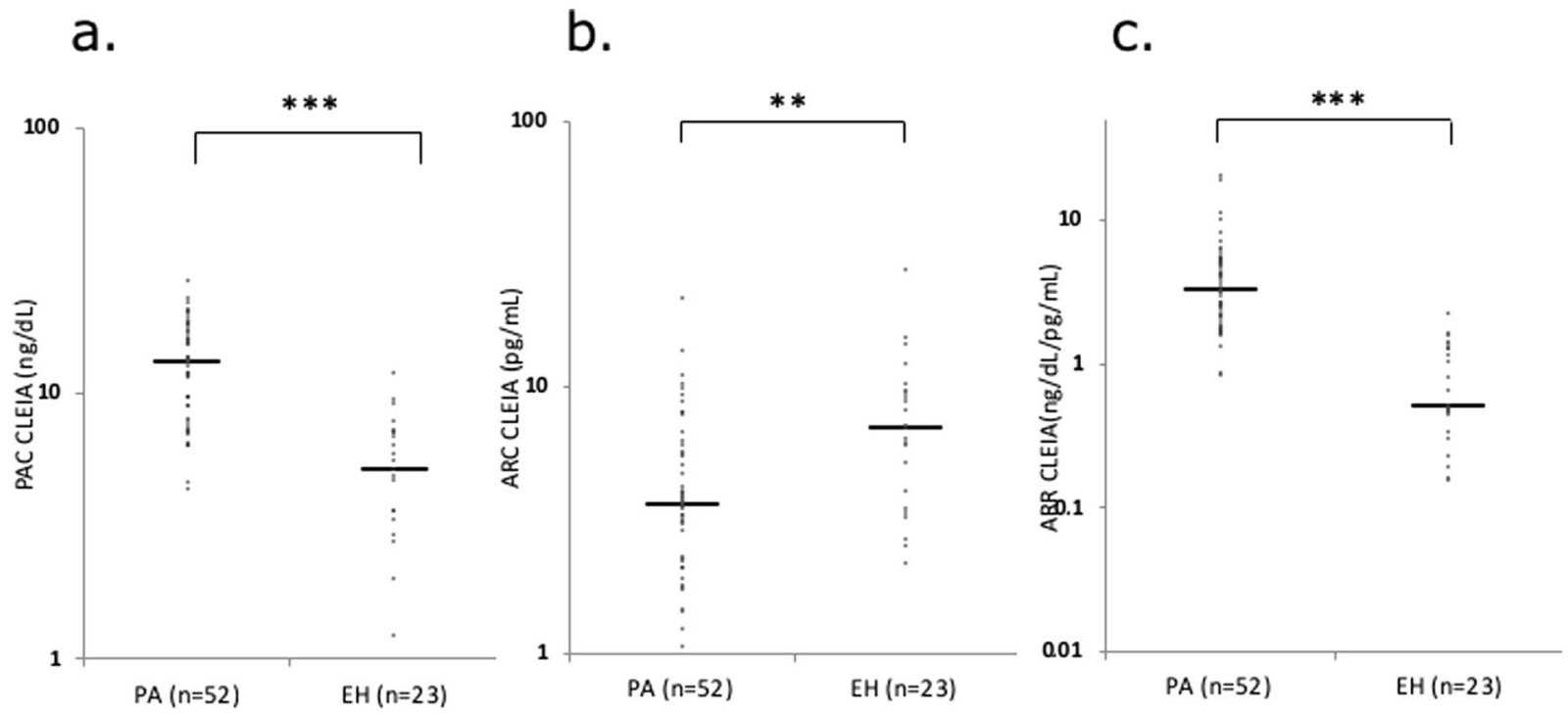

Fig. 4 The plasma aldosterone concentration (PAC) (a), plasma active renin concentration (ARC) (b), and PAC/ARC ratio (ARR) (c) in patients with primary aldosteronism (PA) and those with essential hypertension (EH). $* * * p<0.001, * * p<0.01, * p<0.05$. 


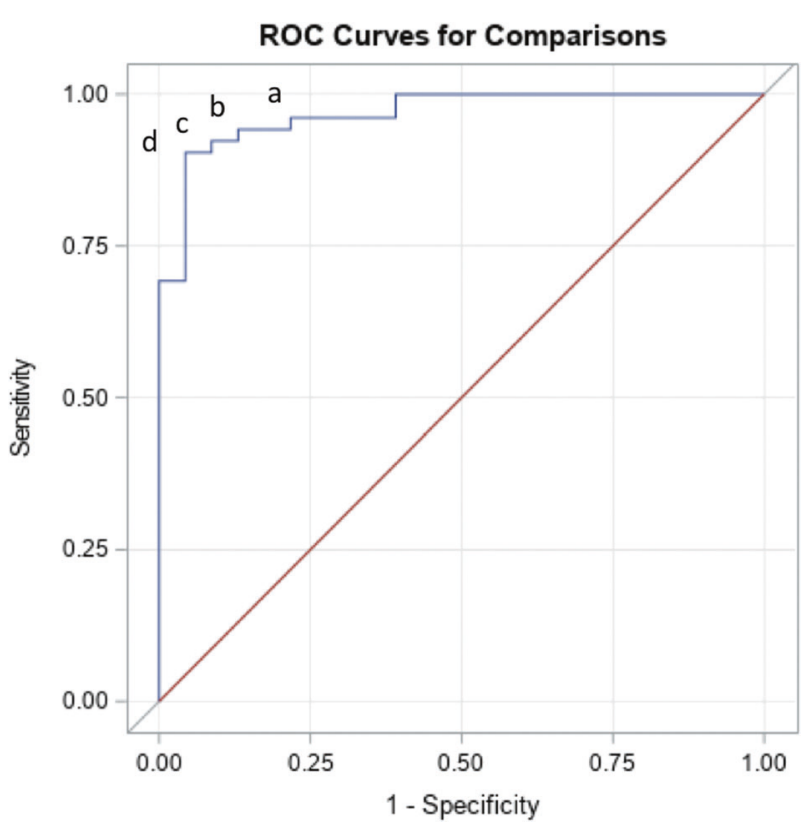

Fig. 5 Receiver operating characteristic analysis of the plasma aldosterone concentration (PAC)/active renin concentration (ARC) ratio (ARR) measured by the chemiluminescent enzyme immunoassay (CLEIA) for screening primary aldosteronism (PA). An ARR cutoff of $1.31 \mathrm{ng} / \mathrm{dl}$ per $\mathrm{pg} / \mathrm{ml}$ had the best sensitivity $(96.2 \%)$ for diagnosing PA, with a specificity of $78.3 \%$ (area under the curve [AUC]: 0.967; 95\% CI: 0.932-1.000) (a). An ARR cutoff of $1.51 \mathrm{ng} /$ dl per $\mathrm{pg} / \mathrm{ml}$ had a sensitivity of $94.2 \%$ and specificity of $87.0 \%$ (b). An ARR cutoff of $1.62 \mathrm{ng} / \mathrm{dl}$ per $\mathrm{pg} / \mathrm{ml}$ had a sensitivity of $92.3 \%$ and specificity of $91.3 \%$ (c). An ARR cutoff of $1.65 \mathrm{ng} / \mathrm{dl} \mathrm{per} \mathrm{pg} / \mathrm{ml}$ had a sensitivity of $90.4 \%$ and specificity of $95.7 \%$ (d).

concentration ranges [13] as well as at higher concentration ranges in adrenal vein samples. In agreement with a previous study [13], our CLEIAs demonstrated small but significant differences from the RIA results, especially at concentrations $<10 \mathrm{ng} / \mathrm{dl}$. However, the CLEIA results were identical to the LC-MS/MS results, even at lower concentrations. Since the optimal cutoff values for confirmatory tests (saline infusion test and fludrocortisone suppression test) exceed $5.0-10 \mathrm{ng} / \mathrm{dl}$ according to the clinical practice guidelines set by the Endocrine Society [9], measurement of relatively low PACs is important in decision making of the confirmatory tests. For PAC measurements, our CLEIA showed a good correlation with LC-MS/MS and good sensitivity and accuracy at concentrations $<10 \mathrm{ng} / \mathrm{dl}$. Thus, it could be a useful tool for the diagnosis of PA.

Our CLEIA for measuring the plasma ARC demonstrated a good correlation with conventional assays. The CLEIA showed a detection sensitivity of $0.04 \mathrm{pg} / \mathrm{ml}$, which was significantly better than that $(5 \mathrm{pg} / \mathrm{ml})$ of IRMA. The detection sensitivity of the ARC is important for determining the ARR, which is universally used for PA screening in patients with hypertension [8-10]. The conventional assay, with a relatively high LoD, has a higher risk of false-negative results in PA screening. The CLEIA, which showed an improved sensitivity, is expected to provide evidence for more detailed cut-off values for screening and accurate diagnosis of PA. Concerning the cut-off value of ARR, Morimoto et al [14]. used the Accuraseed ARC assay to demonstrate that an ARR cut-off of $6.0 \mathrm{ng} / \mathrm{dl}$ per $\mathrm{pg} / \mathrm{ml}$ had $\sim 100 \%$ sensitivity for diagnosing PA. By contrast, Manolopoulou et al [26]. used CLEIA to demonstrate that an ARR cut-off of $1.12 \mathrm{ng} / \mathrm{dL}$ per $\mu \mathrm{IU} / \mathrm{ml}$ (equivalent to $1.87 \mathrm{ng} / \mathrm{dL}$ per $\mathrm{pg} / \mathrm{ml}$ ) had a sensitivity of $98.9 \%$ for diagnosing PA. In agreement with the study by Manolopoulou et al., our ROC analysis of the ARR determined by the novel CLEIA showed the best sensitivity (96.2\%) at a cutoff of $1.31 \mathrm{ng} / \mathrm{dl}$ per $\mathrm{pg} / \mathrm{ml}$. One possible explanation for the discrepancy in cut-off values among studies is the varying degree of hyperaldosteronism among the different PA subtypes. The prevalence of aldosteroneproducing adenoma with a higher PAC than that in idiopathic hyperaldosteronism was $60 \%$ in the study by Morimoto et al [14]. and $13 \%$ in the present study, respectively. Further study is needed to determine the optimal cut-off of ARR for screening PA with consideration of the different PA subtypes.

The present CLEIA is applicable as a standard assay method for measuring the PAC in daily clinical practice, and an automated system with these CLEIAs enables us to obtain PAC and plasma ARC results within $30 \mathrm{~min}$. This significant decrease in measurement time will help improve the efficiency of the PA diagnostic process in the clinic and the success rate of adrenal venous sampling. The novel CLEIAs for PAC and ARC could affect the clinical practice of hypertension and PA.

\section{Limitations}

The present study has several limitations. First, blood sampling in the patients was not optimized in terms of food intake, posture, time of day, or use of antihypertensive medications, which may affect PAC and/or ARC measurements. However, the major aim of the present study was to validate the new CLEIAs and compare their efficacies with those of conventional assays. The sampling conditions likely did not affect the correlations between the assays. Second, an ARR cut-off in the current study is calculated by small sample size. Further systematic studies using larger numbers of samples obtained under standardized conditions are needed to establish the appropriate cut-offs of PAC and ARR for screening PA.

\section{Summary table}

\section{What is known about the topic}

- Radioimmunoassay (RIA) has been used to determine the PAC, ARC, and PRA in clinical practice, although 
various issues with RIAs, such as the usage and disposal of radioisotopic materials, complexity of the manual assay, poor traceability of certified reference materials, and low detection sensitivity at lower concentrations, remain to be resolved.

\section{What this study adds}

- The novel CLEIAs using a fully automated system developed to measure PAC and ARC were characterized by a better detection sensitivity, much shorter measurement time, and lower cost compared with the conventional RIA. These new methods are expected to facilitate the diagnostic process and improve the quality of hypertension and PA clinical practice.

\begin{abstract}
Acknowledgements We thank Maki Kashiwabara and Keiko Umegaki for their technical and secretarial assistance. All of the authors have accepted responsibility for the entire content of this submitted paper and approve its submission.
\end{abstract}

Funding This study was supported in part by grants-in-aid for the Japan Primary Aldosteronism Study and Japan Rare Adrenal Diseases Study of the Practical Research Project for Rare/Intractable Diseases from the Japan Agency for Medical Research and Development (AMED) (JP17ek0109122, JP20ek0109352).

\section{Compliance with ethical standards}

Conflict of interest This study was conducted in part by the research expenses provided by the research contract between NHO Kyoto Medical Center and Fujirebio Inc. (Tokyo, Japan). MN received lecture fees from Daiichi Sankyo Co., Ltd. and Kyowa Kirin Co., Ltd.. The authors declare that no conflicts of interest related to employment, leadership positions, or honoraria.

Publisher's note Springer Nature remains neutral with regard to jurisdictional claims in published maps and institutional affiliations.

Open Access This article is licensed under a Creative Commons Attribution 4.0 International License, which permits use, sharing, adaptation, distribution and reproduction in any medium or format, as long as you give appropriate credit to the original author(s) and the source, provide a link to the Creative Commons license, and indicate if changes were made. The images or other third party material in this article are included in the article's Creative Commons license, unless indicated otherwise in a credit line to the material. If material is not included in the article's Creative Commons license and your intended use is not permitted by statutory regulation or exceeds the permitted use, you will need to obtain permission directly from the copyright holder. To view a copy of this license, visit http://creativecommons. org/licenses/by/4.0/.

\section{References}

1. Shariq OA, Mehta K, Thompson GB, Lyden ML, Farley DR, Bancos I, et al. Primary aldosteronism: does underlying pathology impact clinical presentation and outcomes following unilateral adrenalectomy? World J Surg. 2019;43:2469-76.

2. Yang Y, Reincke M, Williams TA. Prevalence, Diagnosis and Outcomes of Treatment for Primary Aldosteronism Best Pract Res Clin Endocrinol Metab. 2019:101365. https://doi.org/10.1016/j. beem.2019.101365

3. Byrd JB, Turcu AF, Auchus RJ. Primary aldosteronism. Circulation. 2018;138:823-35.

4. Vaidya A, Mulatero P, Baudrand R, Adler GK. The expanding spectrum of primary aldosteronism: implications for diagnosis, pathogenesis, and treatment. Endocr Rev. 2018;39:1057-88.

5. Monticone S, D'Ascenzo F, Moretti C, Williams TA, Veglio F, Gaita F, et al. Cardiovascular events and target organ damage in primary aldosteronism compared with essential hypertension: a systematic review and meta-analysis. Lancet Diabetes Endocrinol. 2018;6:41-50.

6. Ohno Y, Sone M, Inagaki N, Yamasaki T, Ogawa O, Takeda Y, et al. Prevalence of cardiovascular disease and its risk factors in primary aldosteronism: a multicenter study in Japan. Hypertension. 2018;71:530-7.

7. Rossi GP, Maiolino G. Excess arterial damage in hyperaldosteronism. Hypertension. 2019;74:502-4.

8. Nishikawa T, Omura M, Satoh F, Shibata H, Takahashi K, Tamura N, et al. Guidelines for the diagnosis and treatment of primary aldosteronism - The Japan Endocrine Society 2009. Endocr J. 2011;58:711-7.

9. Funder JW, Carey RM, Mantero F, Murad MH, Reincke M, Shibata $\mathrm{H}$, et al. The management of primary aldosteronism: case detection, diagnosis, and treatment: An Endocrine Society clinical practice guideline. J Clin Endocrinol Metab. 2016;101:1889-916.

10. Ujike T, Uemura M, Mukai K, Kozawa J, Otsuki M, Takao T, et al. The latest consensus statement on the clinical practice of primary aldosteronism in Japan. Off J Jpn Assoc Endocr Surg Jpn Soc Thyroid Surg. 2018;35:6-7.

11. Guo Z, Poglitsch M, McWhinney BC, Ungerer JPJ, Ahmed AH, Gordon RD, et al. Aldosterone LC-MS/MS assay-specific threshold values in screening and confirmatory testing for primary aldosteronism. J Clin Endocrinol Metab. 2018;103:3965-73.

12. Japan Health Policy Now, http://japanhpn.org/en/home-1/

13. Nishikawa T, Omura M, Kawaguchi M, Takatsu A, Satoh F, Ito S, et al. Calibration and evaluation of routine methods by serum certified reference material for aldosterone measurement in blood. Endocr J. 2016;63:1065-80.

14. Morimoto R, Ono Y, Tezuka Y, Kudo M, Yamamoto S, Arai T, et al. Rapid screening of primary aldosteronism by a novel chemiluminescent immunoassay. Hypertension. 2017;70:334-41.

15. Nishizono I, Iida S, Suzuki N, Kawada H, Murakami $H$, Ashihara $\mathrm{Y}$, et al. Rapid and sensitive chemiluminescent enzyme immunoassay for measuring reference tumor markers. Clin Chem. 1991;37:1639-44

16. National Institute of Advanced Industrial Science and Technology, Tsukuba, Japan, https://www.nmij.jp/service/C/

17. Mulatero P, Stowasser M, Loh KC, Fardella CE, Gordon RD, Mosso L, et al. Increased diagnosis of primary aldosteronism, including surgically correctable forms, in centres from five continents. J Clin Endocrinol Metab. 2004;89:1045-50.

18. Monticone S, Burrello J, Tizzani D, Bertello C, Viola A, Buffolo F, et al. Prevalence and clinical manifestations of primary aldosteronism encountered in primary care practice. J Am Coll Cardiol. 2017;69:1811-20.

19. Catena C, Colussi G, Nadalini E, Chiuch A, Baroselli S, Lapenna $\mathrm{R}$, et al. Cardiovascular outcomes in patients with primary aldosteronism after treatment. Arch Intern Med. 2008;168:80-85.

20. Milliez P, Girerd X, Plouin PF, Blacher J, Safar ME, Mourad JJ, et al. Evidence for an increased rate of cardiovascular events in 
patients with primary aldosteronism. J Am Coll Cardiol. 2005;45: 1243-8.

21. Mulatero P, Monticone S, Bertello C, Viola A, Tizzani D, Iannaccone A, et al. Long-term cardio- and cerebrovascular events in patients with primary aldosteronism. J Clin Endocrinol Metab. 2013;98:4826-33.

22. Savard S, Amar L, Plouin PF, Steichen O. Cardiovascular complications associated with primary aldosteronism: a controlled cross-sectional study. Hypertension. 2013;62:331-336.

23. Perschel FH, Schemer R, Seiler L, Reincke M, Deinum J, MaserGluth C, et al. Rapid screening test for primary hyperaldosteronism: ratio of plasma aldosterone to renin concentration determined by fully automated chemiluminescence immunoassays. Clin Chem. 2004;50:1650-5.
24. Burrello J, Monticone S, Buffolo F, Lucchiari M, Tetti M, Rabbia $\mathrm{F}$, et al. Diagnostic accuracy of aldosterone and renin measurement by chemiluminescent immunoassay and radioimmunoassay in primary aldosteronism. J Hypertens. 2016;34:920-7.

25. Rossi GP, Ceolotto G, Rossitto G, Seccia TM, Maiolino G, Berton $\mathrm{C}$, et al. Prospective validation of an automated chemiluminescence-based assay of renin and aldosterone for the work-up of arterial hypertension. Clin Chem Lab Med. 2016; 54:1441-50.

26. Manolopoulou J, Fischer E, Dietz A, Diederich S, Holmes D, Junnila R, et al. Clinical validation for the aldosterone-to-renin ratio and aldosterone suppression testing using simultaneous fully automated chemiluminescence immunoassays. J Hypertens. 2015; 33:2500-11. 